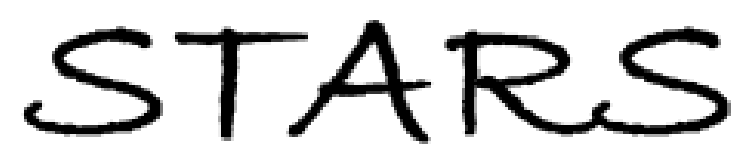

University of Central Florida

STARS

$1-1-1990$

\title{
Analytical And Simulation Models For Real-time Networks
}

Michael Bassiouni

Michael Georgiopoulos

Jack Thompson

Find similar works at: https://stars.library.ucf.edu/istlibrary University of Central Florida Libraries http://library.ucf.edu

This Research Report is brought to you for free and open access by the Digital Collections at STARS. It has been accepted for inclusion in Institute for Simulation and Training by an authorized administrator of STARS. For more information, please contact STARS@ucf.edu.

\section{Recommended Citation}

Bassiouni, Michael; Georgiopoulos, Michael; and Thompson, Jack, "Analytical And Simulation Models For Real-time Networks" (1990). Institute for Simulation and Training. 17.

https://stars.library.ucf.edu/istlibrary/17

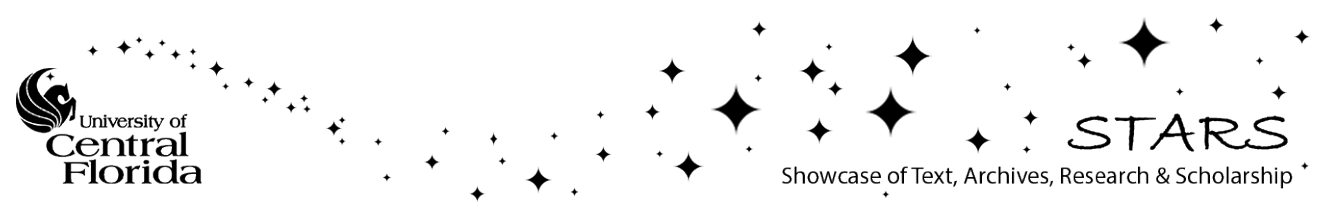




\section{ANALYTICAL AND SIMULATION MODELS FOR REAL-TIME NETWORKS}

M. Bassiouni

Dept. of Computer Science
M. Georgiopoulos

Dept. of Electrical Engineering

University of Central Florida

Orlando, FL 32816 USA

\author{
J.Thompson \\ Inst. for Simulation and Training \\ $1 S T-T R-90-19$
}

\section{ABSTRACT}

In this paper, we present a discussion of analytical and simulation models which have been developed for the purpose of assessing/predicting the performance of local area networks (LANs) used to interconnect distributed, real-time simulation and training devices. Via these models, we examine three different network access protocols; Carrier Sense Multiple Access with Collision Detection (CSMA/CD) [1], Token-Ring [2], and Virtual Token-Passing Bus Access [3]. The implications of the comparison study and insight gained from our research for improving real-time simulation networks are discussed.

\section{INTRODUCTION}

The advent of direct computer-to-computer communications (computer networking) opened the possibility of interconnecting many different types of computer based systems. Until recently, most devices used in Simulation and Training (S\&T), which usually contained an embedded computational resource, operated in a stand alone mode. Today there is major emphasis being alaced on the development of distributed S\&T systems which are networkable. In this context, the im networkable implies that the S\&T systems are capable of communicating (transmitting and receiving), in real-time, information relative to their simulation which can be understood by other networkable devices tied to the network, thus allowing for interaction among the devices.

\section{DISTRIBUTED NETWORKED SIMULATIONS}

In this paper, the terms distributed simulation, simulation networks and real-time training networks are used interchangeably and denote the interconnection of large numbers of S\&T systems via a computer network (e.g., ETHERNET). The devices themselves are herein referred to as S\&T systems, S\&T devices and simulators, interchangeably as well.

The systems are distributed in that they each comprise the appropriate hardware, software and computational resources to allow it to operate in conjunction with, or independent of, other systems on the network. Each S\&T system consists of specialized hardware (host computer, image generator, control input/output devices, etc.) and software which drive the simulation. Typically, the interior of the device bears a resemblance to the vehicle being simulated (armored vehicle, police car, fire truck, etc.). The simulated vehicles are operated identically to actual vehicles. Out-thewindow views are provided via TV monitors which display real-time scenes created by the image generator of the simulator.

In the networked mode of operation, the occupants of two simulated vehicles sitting in front of one another, for example, would be able to visually observe each other's actions. If the driver of one of the simulated vehicles started his engine and began driving, this would be observed by the second vehicle, via the out-the-window view monitors. The driver of the second vehicle could then start his vahicle and pursue the first vehicle, if he wished to do so. The key here is that the actions of each of vehicles is not controlled by the simulation. It is the crew in the simulated vehicle who decides what action will be taken. This is referred to as a free play environment. 
Computer networks are typically used to allow for the sharing of resources (e.g., printers, disk drives, etc.) between multiple computers. In the application of networked S\&T devices, however, the network is used almost exclusively for the communication of data between the simulators. Data the network could consist of information describing the state of the vehicle (e.g., location, vientation, etc.), as well as other information involved in the simulation such as digitized voice communications data.

In this application, the real-time performance of the network is of utmost importance. If data delays through the network become too large, the effectiveness of a real-time S\&T system may be compromised due to the time-critical response requirements in the simulation of true-to-life, actionresponse training scenarios.

The Institute for Simulation and Training at the University of Central Florida (IST-UCF) has established a Network and Communications Laboratory dedicated to performing research for the purpose of enhancing the networking capabilities of these types of S\&T distributed simulations. This laboratory houses a number of real-time, networkable simulators and is the center of several research activities dealing with different aspects of S\&T device networking.

\section{NETWORK SIMULATION MODELS}

There are a wide variety of commercially available LANs which might be used to interconnect S\&T devices [1-6]. In our study we chose to investigate three LAN implementations, namely, ETHERNET, TOKEN-RING and Virtual Token-Passing Bus Method. Of these, the ETHERNET and the Virtual Token-Passing Bus Method are implemented via a bus topology where all nodes are connected to the network medium in a parallel fashion, while the TOKEN-RING is implemented via a loop or ring topology where the network medium actually passes through each node in a serial fashon. Further, both the TOKEN-RING and the Virtual Token-Passing Bus Method are noncontention access protocols in that the nodes on the net do not compete for network access vileges. The ETHERNET, on the other hand, is contention access protocol. A brief discussion reach of the three LAN implementations is given below.

(NOTE: For the purposes of our research, a specific implementation of the Virtual Token-Passing Bus Method referred to as Generalized Broadcast Recognizing Access Method (GBRAM) was chosen for study. This acronym (GBRAM) will appear throughout the remainder of this report.)

\section{ETHERNET}

ETHERNET is an implementation of the CSMA/CD class of protocols [1] based on a bus topology. Devices, or nodes, connected via ETHERNET gain access to the bus network by the following sequence. If a node has data to transmit, it first monitors the network bus to determine whether any other transmissions from other nodes are in progress. If the node senses traffic on the network, it withholds the transmission of its data until there is no traffic on the network. At this time, the node then waits an additional amount of time, referred to as the inter-frame gap, before attempting transmission of its data. This interframe gap wait time is an extra precaution to assure the network is truly idle. Now the node will begin transmitting its data and will simultaneously monitor the network in order to determine whether its transmission is interfering (colliding) with transmitted data from other nodes. If a collision is detected, all nodes involved in the collision send out a jam signal which alerts all nodes on the network that a collision has occurred. The colliding nodes then wait (back-off) for a randomly calculated amount of time before reattempting their transmissions. Since each node calculates its own back-off time, chances for subsequent collisions are minimized. A maximum of fifteen retransmissions attempts will be made, after which the data will be discarded. The problem of collisions is directly related to the number of nodes on the network and the amount of data being generated by each node. 
TOKEN-RING

TOKEN-RING is an implementation of the Token Passing class of protocols $[2,7]$ based on a ring (loop) topology. In the TOKEN-RING, nodes are arranged in a serial ring configuration such that the twork channel actually passes through each node. The token is a unique sequence of data which circulates around the network, from one node to the next. Contention for transmission is resolved by stipulating that only the node currently in possession of the token is allowed to transmit a frame, or sequence of frames, onto the network. After a node has completed its transmission, the token is passed on to the next (downstream) node which then gains the privilege to transmit its data. While the overhead associated with token management is greater than ETHERNET's overhead, it will be shown that at high traffic loads the overhead more than pays for itself.

\section{GENERALIZED BROADCAST RECOGNIZING ACCESS METHOD}

The Generalized Broadcast Recognizing Access Method (GBRAM) [3] is a contention free protocol implemented on a bus topology. Its contention free operation is based on a decentralized scheduling function which provides each node access to the network at a unique instant in time. Under GBRAM, each node on the network perceives the channel state as consisting of cycles of scheduling and transmission periods. Typically, the end of a transmission period signifies the beginning of a scheduling period and the end of a scheduling period where there was no transmission signifies the beginning of the subsequent scheduling period. The scheduling function determines the next node which will be allowed access to the network. As soon as the node starts its transmission, the scheduling period is terminated. A new scheduling period will begin only after the end of the current transmission period.

\section{NETWORK PROTOCOL SIMULATION MODELS}

Detailed simulation programs were written to assess/predict the performance of the ETHERNET, TOKEN-RING and GBRAM networks when used to interconnect large numbers of networked S\&T devices. The simulation models for both the bus and ring network topologies are written in oncurrent-C which is an extension of the C programming language with concurrent programming facilities based on the rendezvous concept. The powerful synchronization and concurrency aspects of Concurrent-C [8] have provided us with a notationally convenient and conceptually elegant tool for modeling the parallel activities of LAN nodes and the underlying networking layer.

Typically, up to eight simulators can be connected to the network bus at a single point via a multiport transceiver. Descriptions of of the Concurrent-C process types used in the simulation of the bus network topology are given below.

The Simnode process is used to model the data generation characteristics of individual simulators. This process is the source of local traffic and is capable of generating traffic with various characteristics (trace data or randomly generated data inter-arrival times).

The Busnode process is used to model the point of contact of each node with the network bus. Functionally, upon receiving a data transmission request from a Simnode process, Busnode attempts to fulfill the request based on the access protocol method implemented by the LAN being modeled.

The Lserver and Rserver processes are used to simulate the propagation delay and control the flow of data traversing the network from right to left (Lserver) and left to right (Rserver) between Busnodes.

A Scheduler process is used to control the sequencing of the entire simulation.

Vescriptions of of the Concurrent-C process types used in the simulation of the ring network topology are given below. 
The Simnode process is used, once again, to model the data generation characteristics of the 'individual simulators on the network. The Ringnode process, which is analogous to the Busnode process of the bus network, monitors the ring traffic and implements the token based network ccess protocol method. The Server process is used to simulate the flow of traffic between nodes and the propagation delay of the network. As in the bus model, a Scheduler process is used to control the sequencing of the entire ring network simulation.

\section{NETWORK SIMULATED PERFORMANCE OBSERVATIONS}

The main purpose of our study was to investigate the suitability of implementing a simulation network using the ETHERNET, TOKEN-RING and GBRAM protocols. Suitability, in this context, can be taken to mean the real-time performance of a network where the majority of its traffic is made up of broadcast type data, which is intended for all nodes on the network, as opposed to point-to-point data traffic where one node sends data only to one other node. This broadcast type traffic is characteristic of simulation networks.

For all network performance simulations, we have made the assumptions that the bandwidth,or capacity, of the network channels are identical and set it to be $10 \mathrm{Mbits} / \mathrm{sec}$.

\section{ETHERNET}

The numerous simulation tests we have conducted show that the throughput of simulation networks implemented via ETHERNET reach a maximum of about $60-70 \%$ of the network bandwidth. For a $10 \mathrm{Mbits} / \mathrm{sec}$ ETHERNET, this amounts to a throughput of approximately 6-7 Mbits/sec. The saturation of the throughput is primarily due to the excessive rate of collisions between nodes attempting to transmit their data simultaneously, which is characteristic under high traffic conditions. Our tests also indicate that as collisions begin to increase, there is a corresponding increase in the delay experienced by each data packet transmitted through the network due to the overhead involved in resolving collisions. Additionally, there are instances where data is lost due to xceeding the limit of retransmission attempts. Eventually, as the level of traffic exceeds the 6-7 ribits/sec level, the performance of ETHERNET rapidly collapses causing packet delays to become so large that they are no longer acceptable for real-time simulations.

\section{TOKEN-RING}

Simulation tests for the TOKEN-RING network configuration have consistently produced results indicating a maximum throughput in the range of $90-95 \%$ of the network bandwidth, or about 9-9.5 Mbits/sec. (the 5-10\% loss of network bandwidth is due to token-passing across the network). Due to its collision-free nature, the TOKEN-RING network does not experience degradation at high traffic levels. And since the circulation of the token operates in a deterministic manner, a maximum packet delay can be guaranteed for a given number of nodes on the network.

\section{GBRAM}

The GBRAM network utilizes the the same bus topology as the ETHERNET implementation. Our simulation results for the GBRAM network configuration indicate a maximum throughput of nearly $100 \%$ of the network bandwidth (almost $10 \mathrm{Mbits} / \mathrm{sec}$ ). This very high throughput is realized because only a very small portion of the network bandwidth is sacrificed for network management (e.g., virtual token passing). Due to its collision-free nature, the GBRAM network does not experience degradation at high traffic levels. And like TOKEN-RING, a maximum packet delay can be guaranteed for a given number of nodes connected by a GBRAM network due to its deterministic nature in scheduling transmission instances.

\section{CONCLUSIONS}

Herein we have discussed various aspects of our research involving the networking of distributed imulation and Training Devices. Our focus has been directed towards the requirements of Local nrea Networks (LANs) utilized in this application. Of particular interest in these types of LAN 
implementations is the real-time performance requirements brought on by the simulation of true-tolife, action requiring training scenarios.

Our LAN simulations for the ETHERNET, TOKEN-RING and GBRAM network protocols indicate that ontention-free protocols (TOKEN-RING and GBRAM) demonstrate superior performance over contention protocols (ETHERNET) for simulation networks with high traffic loads (i.e., 65-90\% of the network bandwidth) in the areas of throughput and packet delay. However, under light to medium traffic loads, the ETHERNET protocol exhibits excellent throughput and delay performance.

We would like to note that because of the page limitation of this publication, we were unable to present any discussion of the analytical portion of our research work, which has been primarily focused on the greedy-node problem associated with CSMAVCD types of networks. Details of this research can be found in the document entitled Performance Evaluation of Local Area Networks for Real-TIme Simulation avaliable from the authors.

\section{ACKNOWLEDGEMENTS}

This work has been supported by U.S. Army's Program Manager for Training Devices (PM TRADE) and the Defense Advanced Projects Research Agency (DARPA) under Broad Agency Announcement \#88-01, contracts N61339-88-G-002 Order 008 and N61339-89-C-0043. The views and conclusions herein are those of the authors and do not represent the official policies of the funding agencies, the Institute for Simulation and Training, or the University of Central Florida.

The authors would like to thank J. Cadiz, M. Chiu, N. Christou and E. Stadler for their assistance during the course of this project.

\section{REFERENCES}

[1] ANSI/IEEE - International Standard 8802/3 "Carrier sense multiple access with collision detection (CSMA/CD) access method and physical layer specificaion". IEEE Computer Society Press, 1985.

[2] ANSI/IEEE - International Standard 8802/5 "Token ring access method" IEEE Computer Society Press, 1985.

[3] Liu, T.; Li, L and Franta, W. "A decentralized conflict-free protocol, GBRAM, for large scale networks" Proc. Computer Network Symposium, December 1981, pp. 39-54.

[4] Kolnik, I and Garodnick, J. "First FDDI local area network" Proc. 12th IEEE Conference on Local Computer Networks, 1987, pp. 7-11.

[5] Stallings, W. "Local networks" ACM Computing Surveys, Vol. 16, No. 1, March 1984, pp. 3-42.

[6] Tanenbaum, A. "Network protocols" ACM Computing Surveys, Vol. 13, No. 4, Dec. 1981, pp. 453-490.

[7] ANSI/IEEE - International Draft Standard 8802/4 "Token-passing bus access method" IEEE Computer Society Press, 1985.

[8] N. Gehani and Roome, W. "Concurrent C" Technical Report, AT\&T Bell Laboratories, 1986. 